\title{
ON THE SINGULARITIES OF CERTAIN NONLINEAR PARTIAL DIFFERENTIAL EQUATIONS
}

\author{
PATRICIO AVILES and ALLEN WEITSMAN*
}

0. Introduction. In this paper we shall study some aspects of the singularities of equations $-\Delta_{p} u=f(x, u)$ where $\Delta_{p} u \equiv \operatorname{div}\left(|\nabla u|^{p-2} \nabla u\right)(p>1)$ is the so-called $p$-Laplacian, and $f$ a continuous function subject to certain growth restrictions. Equations of this type have been studied in connection with a variety of problems (cf. references in [10]).

Theorem 1 of this paper complements a recent series of works on removable singularities [3], [1], [12], [11]. In [11] the following is proved:

Theorem A. Let $\Omega$ be an open set in $\boldsymbol{R}^{n}, q \in \Omega$, and $\Omega^{\prime}=\Omega-\{q\}$. Suppose $1<p<n$, that $f$ is a continuous real function on $\Omega \times \boldsymbol{R}$ satisfying

$$
\liminf _{r \rightarrow \infty} \frac{f(x, r)}{r^{n(p-1) /(n-p)}}>0, \quad \limsup _{r \rightarrow-\infty} \frac{f(x, r)}{|r|^{n(p-1) /(n-p)}}<0
$$

uniformly in $\Omega$, that $u \in W_{\mathrm{loc}}^{1, p}\left(\Omega^{\prime}\right) \cap L_{\mathrm{loc}}^{\infty}\left(\Omega^{\prime}\right)$, and $\Delta_{p} u \in L_{\mathrm{loc}}^{1}\left(\Omega^{\prime}\right)$ (in the sense of distributions). Then if $u$ is a solution of

$$
-\Delta_{p} u+f(x, u)=0
$$

in $\mathscr{D}^{\prime}\left(\Omega^{\prime}\right)$, there exists a locally Hölder continuous function $\tilde{u}$, defined in all of $\Omega$, which coincides with $u$ a.e. in $\Omega$ and satisfies (2) in $\mathscr{D}^{\prime}(\Omega)$.

We have

Theorem 1. If in Theorem A we take $p=n$ and replace condition (2) by

$$
\liminf _{r \rightarrow \infty} \frac{f(x, r)}{e^{r \delta}}>0, \quad \limsup _{r \rightarrow-\infty} \frac{f(x, r)}{e^{|r| \delta}}<0
$$

for some fixed $\delta>1$, then the conclusions of Theorem A again hold.

In $\S 5$ we shall discuss examples to show $\delta>1$ in (3) is essential.

In [10], interior estimates are derived for functions $u$ satisfying

$$
-\Delta_{p} u+f(u) \leqq 0
$$

*) Research of the authors supported by the National Science Foundation. 
in the case of "weak diffusion for large intensities":

$$
\int_{0}^{\infty} d r\left(\int_{0}^{r} f(s) d s\right)^{-1 / p}<\infty
$$

For $p=2$, conditions of this type have been used in connection with nonexistence of entire solutions by numerous authors [14], [13], [6], [7], [2]. In our next result we study this effect for the $p$-Laplacian.

Theorem 2. Let $f(s)$ be a positive nondecreasing locally Lipschitz function defined on $\boldsymbol{R}$ and satisfying (5).

(A) If $1<p$ then (4) has no subsolutions $u$ with $u \in W_{\mathrm{loc}}^{p}\left(\boldsymbol{R}^{n}\right) \cap L_{\mathrm{loc}}^{\infty}\left(\boldsymbol{R}^{n}\right)$ and $\Delta_{p} u \in$ $L_{\mathrm{loc}}^{1}\left(\boldsymbol{R}^{n}\right)$ (in the sense of distributions).

(B) If $1<p<n$ and $S$ is any compact subset of $\boldsymbol{R}^{n}$ then there are no subsolutions $u$ of (5) with $u \in W_{\mathrm{loc}}^{1, p}\left(\boldsymbol{R}^{n}-S\right) \cap L_{\mathrm{loc}}^{\infty}\left(\boldsymbol{R}^{n}-S\right)$ and $\Delta_{p} u \in L_{\mathrm{loc}}^{1}\left(\boldsymbol{R}^{n}-S\right)$ (in the sense of distributions).

I. Preliminary lemmas. There are general comparisons theorems which cover the $p$-Laplacian. We require only a very simple version (cf. $[11 ;$ p. 5]).

Lemma A. In a region $\Omega \subseteq \boldsymbol{R}^{n}$ suppose $u, v \in W_{\mathrm{loc}}^{1, p}(\Omega) \cap L_{\mathrm{loc}}^{\infty}(\Omega) \quad(1<p)$, $\Delta_{p} u, \Delta_{p} v \in L_{\mathrm{loc}}^{1}(\Omega)$ (in the sense of distributions) and $(u-v)^{+} \in W_{0}^{1, p}(\Omega)$. If $g$ is a nondecreasing function on $\boldsymbol{R}$ and

then $u \leqq v$ a.e. in $\Omega$.

$$
\begin{aligned}
& -\Delta_{p} u+g(u) \leqq 0 \quad \text { in } \mathscr{D}^{\prime}(\Omega) \\
& -\Delta_{p} v+g(v) \geqq 0 \quad \text { in } \mathscr{D}^{\prime}(\Omega),
\end{aligned}
$$

Proof. Let $\Psi \in \mathscr{C}^{1}(\boldsymbol{R})$ be bounded, vanishing on $(-\infty, 0]$, and strictly increasing on $[0, \infty)$. Then, since $\Psi(u-v) \in W_{0}^{1, p}(\Omega)$, (1.1) implies

$$
\int_{\Omega}\left(|\nabla u|^{p-2} \nabla u-|\nabla v|^{p-2} \nabla v\right) \cdot(\nabla u-\nabla v) \Psi^{\prime}(u-v) d x \leqq \int_{\Omega}(g(v)-g(u)) \Psi(u-v) d x .
$$

Now, $p>1$ so $\left(|\nabla u|^{p-2} \nabla u-|\nabla v|^{p-2} v\right) \cdot(\nabla u-\nabla v) \geqq 0$, and $\Psi^{\prime} \geqq 0$ as well. Thus, it follows from the Poincaré lemma that $u \leqq v$ a.e. on $\Omega$.

Lemma 1. Let $\Omega \subseteq \boldsymbol{R}^{n}$ be a region and $q \in \Omega, \Omega^{\prime}=\Omega-\{q\}$. Suppose $u \in W_{\mathrm{loc}}^{1, n}\left(\Omega^{\prime}\right) \cap L_{\mathrm{loc}}^{\infty}\left(\Omega^{\prime}\right)$ and $\Delta_{n} u \in L_{\mathrm{loc}}^{1}\left(\Omega^{\prime}\right)$ (in the sense of distributions in $\left.\Omega^{\prime}\right)$. If for some constants $a>0, C \geqq 0, \delta>1$

$$
-\Delta_{n} u+e^{a u^{\delta}} \leqq C
$$

a.e. on $\{x \in \Omega: u(x) \geqq 0\}$, then $u^{+} \in L_{\mathrm{loc}}^{\infty}(\Omega)$. 
Proof. We may take $q=0$ and $\Omega=\{|x|<\varrho\}$ for some $0<\varrho<1$. Given $x_{0}$ such that $0<\left|x_{0}\right|<\varrho / 2$ we define, for $\delta \geqq 1$

$$
V(x)=V_{x_{0}}(x)=\left(\log \frac{1}{R^{n /(n-1)}-\left|x-x_{0}\right|^{n /(n-1)}}\right)^{1 / \delta}\left(R=\frac{\left|x_{0}\right|}{2},\left|x-x_{0}\right|<R\right) .
$$

Now $V$ as defined is radial about $x_{0}$, and hence writing $r=\left|x-x_{0}\right|$ and taking differentiations with respect to $r$ we have $(n-1) \dot{V}^{n-2}(\ddot{V}+(1 / r) \dot{V})=\Delta_{n} V$. Now,

$$
\begin{gathered}
\dot{V}=\frac{n}{\delta(n-1)}\left(\log \frac{1}{R^{n /(n-1)}-r^{n /(n-1)}}\right)^{(1 / \delta)-1}\left(\frac{r^{1 /(n-1)}}{R^{n /(n-1)}-r^{n /(n-1)}}\right)= \\
=\frac{n}{\delta(n-1)} V^{1-\delta} e^{V \delta} r^{1 /(n-1)} \\
\ddot{V}=\frac{n}{\delta(n-1)}\left(\frac{n\left(\delta^{-1}-1\right)}{(n-1)}\left(\log \frac{1}{R^{n /(n-1)}-r^{n /(n-1)}}\right)^{(1 / \delta)-2}\left(\frac{r^{1 /(n-1)}}{R^{n /(n-1)}-r^{n /(n-1)}}\right)^{2}\right. \\
\quad+\left(\log \left(\frac{1}{R^{n /(n-1)}-r^{n /(n-1)}}\right)^{(1 / \delta)-1} \times\right. \\
\left.\times\left(\frac{r^{(2-n) /(n-1)}}{(n-1)\left(R^{n /(n-1)}-r^{n /(n-1)}\right)}+\frac{n r^{2 /(n-1)}}{(n-1)\left(R^{n /(n-1)}-r^{n /(n-1)}\right)^{2}}\right)\right) \\
\leqq \frac{n}{n-1} V^{1-\delta}\left(\frac{e^{V \delta} r^{(2-n) /(n-1)}}{n-1}+\frac{n e^{2 V \delta}}{n-1}\right) \leqq 2\left(\frac{n}{n-1}\right)^{2} V^{1-\delta} e^{2 V \delta} r^{(2-n) /(n-1)} .
\end{gathered}
$$

Thus,

$$
\begin{aligned}
\Delta_{n} V \leqq & \frac{n^{n}}{(n-1)^{n-1}} V^{(1-\delta)(n-2)} e^{(n-2) V \delta} r^{(n-2) /(n-1)}\left(2 V^{1-\delta} e^{2 V \delta} r^{(2-n) /(n-1)}\right. \\
& \left.+V^{(1-\delta)} e^{V \delta} r^{(2-n) /(n-1)}\right) \leqq \frac{4 n^{n}}{(n-1)^{n-1}} V^{(1-\delta)(n-1)} e^{n V^{\delta}} .
\end{aligned}
$$

Let $v=a^{-\delta-1} n V$. Then, from (1.3) it follows that

$$
\Delta_{n} v \leqq \frac{4 n^{2 n-1} a^{(1-n) / \delta}}{(n-1)^{n-1}} V^{(1-\delta)(n-1)} e^{n V \delta} \leqq \frac{4 n^{2 n-1} a^{(1-n) / \delta}}{(n-1)^{n-1}} V^{(1-\delta)(n-1)} e^{a v \delta} .
$$

From (1.2) we find that for $x_{0}$ and hence $R$ sufficiently small, $V$ and $v$ can be made arbitrarily large. It then follows from (1.4) that there exists $R_{0}=R_{0}(n, \delta)$ such that for all $x_{0}$ with $0<\left|x_{0}\right| \leqq R_{0}$, the corresponding $v(x)=v_{x_{0}}(x)$ all satisfy

$$
\Delta_{n} v \leqq e^{a v \tilde{s}}-C .
$$


Comparing (1.1) and (1.5) and taking account of the fact that $v$ is infinite for $x$ on $\left|x-x_{0}\right|=\left|x_{0}\right| / 2$ we may apply Lemma $\mathrm{A}$ and obtain for a.e. $x_{0}$ such that $0<\left|x_{0}\right| \leqq R_{0}$ the estimate

$$
u\left(x_{0}\right) \leqq v\left(x_{0}\right)=a^{-\delta^{-1}} n V\left(x_{0}\right)=a^{-\delta^{-1}} n\left(\frac{n}{n-1} \log \frac{2}{\left|x_{0}\right|}\right)^{1 / \delta} .
$$

To complete the proof we show that (1.1) and (1.6) imply that $u^{+} \in L^{\infty}\left(|x|<R_{0}\right)$.

If we replace $u$ by $U=\varkappa u(x>1)$ then $\Delta_{n} U=\chi^{n-1} \Delta_{n} u \geqq \chi^{n-1} e^{a \varkappa-\delta} U^{\delta}-C$. Thus, we may fix $x$ sufficiently large so that a.e. for $0<|x|<R_{0}$

$$
\Delta_{n} U \geqq e^{\tilde{a} U^{\delta}} \quad(\tilde{a}>0)
$$

and

$$
U(x) \leqq x a^{-\delta^{-1}} n\left(\frac{n}{n-1} \log \frac{2}{|x|}\right)^{1 / \delta}
$$

Thus, we may choose $M>0$ such that for any $\varepsilon>0$,

$$
\left(U-M-\varepsilon \log (1 /|x|)^{+} \in W_{0}^{1, n}\left(|x|<R_{0}\right) .\right.
$$

With $\Delta_{n}(\varepsilon \log (1 /|x|))=0$ along with (1.7) we may again apply Lemma $\mathrm{A}$ and conclude that $U(x) \leqq M+\varepsilon \log (1 /|x|)$ a.e. in $|x|<R_{0}$. Since $\varepsilon>0$ was arbitrary and $u=x^{-1} U$ the proof is complete.

II. Proof of Theorem 1. Theorem 1 now follows in a standard way (cf. [11:p. 9]) from Lemma 1. Briefly, it suffices from [8; p. 269] to show that $u$ is a weak locally $L^{\infty}$ solution of (2), since $f$ is continuous. Assume $q=0$. Now (3) and Lemma 1 imply that $u \in L_{\mathrm{loc}}^{\infty}(\Omega)$. Let $0 \leqq \eta \in \mathscr{C}_{0}^{\infty}(\Omega)$ and $\zeta_{m} \in \mathscr{C}^{\infty}(\Omega)$ such that

$$
\zeta_{m}(x)=\left\{\begin{array}{ll}
0 & \text { if } \quad|x|<\frac{1}{2 m} \\
1 & \text { if } \quad|x|>\frac{1}{m}
\end{array} \quad 0 \leqq \zeta_{m} \leqq 1,|\nabla \zeta| \leqq c m .\right.
$$

Then, if $\Lambda$ is a relatively compact neighborhood of the origin in $\Omega$, and containing supp $\eta$

$$
\int_{\Lambda} \zeta_{n}|\nabla u|^{n-2} \nabla u \cdot \nabla \eta d x+\int_{\Lambda} \eta|\nabla u|^{n-2} \nabla u \cdot \nabla \zeta_{n} d x+\int_{\Lambda} f(x, u(x)) \zeta_{m} \eta d x=0,
$$

so it suffices to show that

$$
\int_{\Omega} \eta|\nabla u|^{n-2} \nabla u \cdot \nabla \zeta_{m} \rightarrow 0 \quad \text { as } \quad m \rightarrow \infty .
$$


We must show first that $|\nabla u| \in L_{\text {loc }}^{n}(\Omega)$. Now, $u \in W_{\text {loc }}^{1, n}\left(\Omega^{\prime}\right)$, so the rest follows from

$$
\begin{aligned}
& \left|\int_{\Lambda} f(x, u(x)) \zeta_{m}^{n} u d x\right|=\left.\left|\int_{\Lambda}\right| \nabla u\right|^{n-2} \nabla u \cdot\left(\zeta_{m}^{n} u\right) \mid \\
& =\left.\left|\int_{\Lambda}\right| \nabla u\right|^{n} \zeta_{m}^{n} d x+n \int_{\Lambda} \zeta_{m}^{n-1}|\nabla u|^{n-2} \nabla u \cdot \nabla \zeta_{m} \mid \\
& \geqq\left\|\zeta_{m}|\nabla u|\right\|_{L^{n}(\Lambda)}^{n}-n\left\|\zeta_{m}|\nabla u|\right\|_{L^{n}(\Lambda)}^{n-1}\left\|u \nabla \zeta_{m}\right\|_{L^{n}(\Lambda)}
\end{aligned}
$$

since the left-hand side remains bounded, as well as the term $\left\|u \nabla \zeta_{m}\right\|_{L^{n}(\Lambda)}$, as $m \rightarrow 0$.

Thus, returning to $(2.1)$ we have

$$
\left.\left|\int_{\Omega} \eta\right| \nabla u\right|^{n-2} \nabla u \cdot \nabla \zeta_{m} d x \mid \leqq\left(\int_{1 / 2 m<|x|<1 / m}|\nabla u|^{n}\right)^{(n-1) / n}\left(\int_{1 / 2 m<|x|<1 / m}\left|\eta \nabla \zeta_{m}\right|^{n}\right)^{1 / n}
$$

which tends to zero as $m \rightarrow \infty$.

III. Proof of Theorem 2(A). The proof is based on the following

Lemma 2. If $f$ satisfies (5) then the ordinary differential equation

$$
|\dot{v}|^{(p-2)}\left((p-1) \ddot{v}(r)+\frac{(n-1)}{r} \dot{v}(r)\right)=f(v(r))
$$

has solutions for $r>0$ with the following properties

$$
\left\{\begin{array}{l}
\dot{v}(0)=0, v(0)=a, a \in \boldsymbol{R}, \dot{v}>0 \text { if } r>0 \\
v(r) \rightarrow \infty \text { as } r \rightarrow r_{0} \text { with } r_{0}<\infty, v \in \mathscr{C}^{2}\left(0, r_{0}\right) .
\end{array}\right.
$$

Proof. We first construct a solution of (3.1) with $a=0$. For, we consider the formula

$$
v(r)=\int_{0}^{r}\left(\frac{1}{s^{n-1}} \int_{0}^{s} t^{n-1} f(v(t) d t)^{1 /(p-1)}\right) d s .
$$

Applying to (3.3) the Picard iteration process with $v_{0} \equiv 0$ we obtain a local solution of

$$
\dot{v}^{(p-2)}\left((p-1) \ddot{v}(r)+\frac{(n-1)}{r} \dot{v}(r)\right)=f(r)
$$

with the properties

$$
v(0)=0, \dot{v}(0)=0, \dot{v}(r)>0 \text { and } v \in \mathscr{C}^{2} \text { whenever defined for } r>0 .
$$

If there is $r_{0}$ as in (3.2) we are done. Otherwise, with the usual existence and uniqueness theorems, $v$ may be continued to a solution of (3.4) with the properties in (3.5) in a larger interval. Since $\dot{v}(r)>0$, this local process may be repeated indefinitely unless there is $r_{0}<\infty$ such that $v\left(r_{0}\right)=\infty$. We now prove that (5) forces this situa- 
tion. We observe that (3.4) can be written as

$$
\int_{0}^{r}\left(\dot{v}^{(p-1)} r^{(n-1)}\right)_{r} d r=\int_{0}^{r} f(v) r^{(n-1)} d r .
$$

Since $f$ and $v$ are nondecreasing, (3.6) implies

$$
(p-1) \ddot{v} \geqq \dot{v} / r .
$$

Substituting (3.7) into (3.8) we have

$$
(p-1) n \ddot{v} \dot{v}^{(p-2)} \geqq f(v) \text {. }
$$

Multiplying by $\dot{v}$ and integrating we get that there is a positive constant $C$ such that

$$
\dot{v}\left(\int_{0}^{v(r)} f(s) d s\right)^{-1 / p}>C .
$$

From here we obtain

$$
\int_{0}^{v(r)}\left(\int_{0}^{t} f(s) d s\right)^{-1 / P} d t>C r
$$

From (5) and (3.8) it follows that there exists $r_{0}<\infty$ such that $v(r) \rightarrow \infty$ as $r \rightarrow r_{0}$.

To obtain solutions for arbitrary $a$ we consider

$$
|\dot{v}|^{(p-2)}((p-1) \ddot{v}(r)+((n-1) /(r) \dot{v}(r))=g(v(r))
$$

with $g(t)=f(t+a)$. Since $g(t)$ also satisfies (5) there is $v$ satisfying (3.1), (3.2) with $a=0$. Now it is enough to take $\bar{v}=v+a$. This completes the proof of the lemma.

To prove Theorem 2(A) we observe that $v(r)$ is a radial solution of $\Delta_{p} u=f(u)$ if and only if $v$ satisfies (3.1) for $r>0$. On the other hand it is easy to see that if $v$ satisfies (3.1) and (3.2) then $v$, in fact, satisfies

$$
\Delta_{p} v=f(v) \text { in } \mathscr{D}^{\prime}\left(B\left(0, r_{0}\right)\right)
$$

with $B\left(0, r_{0}\right)=\left\{x:\|x\|<r_{0}\right\}$. Therefore, from Lemma $A$ it now follows that

$$
u(x) \leqq v(x) \text { in } B\left(0, r_{0}\right) .
$$

Taking in (3.2), $a=\operatorname{ess~inf}_{x \in \boldsymbol{B}\left(0, r_{0}\right)} u(x)$ we get a contradiction. Therefore, Theorem 2(A) is now complete.

IV. Proof of Theorem 2 (B). Given $-\infty<\alpha<\infty, \beta \neq 0$, the ordinary differential equation

$$
|\dot{v}(r)|^{p-2}\left((p-1) \ddot{v}(r)+\frac{(n-1)}{r} \dot{v}(r)\right)=f(v)
$$

can be solved uniquely with initial data

$$
v(1)=\alpha, \quad \dot{v}(1)=\beta
$$

and continued in each direction. 
Now, for $\dot{v}>0$ (4.1) is the same as $\left(\dot{v}(r)^{p-1} r^{n-1}\right)_{r}=r^{n-1} f(v(r))$ and for $\dot{v}<0$ (4.1) becomes $\left((-\dot{v}(r))^{p-1} r^{n-1}\right)_{r}=-r^{n-1} f(v(r))$. Thus, we may continue $v$ to the left and right from $r=1$ until either $\dot{v}=0$ or $v=\infty$. If neither of these occurs on a side of $r=1$, the continuation proceeds indefinitely in that direction.

We wish to show first that, given numbers $\alpha, M$ there exists $\beta=\beta(\alpha, M)$ such that the solution $u$ of (4.1) with initial condition (4.2) satisfies

$$
v\left(\frac{1}{2}\right) \geqq M .
$$

In fact, with $\beta<0$ and $r<1$,

$$
r^{n-1}(-\dot{v}(r))^{p-1}=(-\beta)^{p-1}+\int_{r}^{1} t^{n-1} f(v(t)) d t
$$

which shows $\dot{v}$ stays negative. Hence from (4.1) we have $(-\dot{v})^{p-2}(p-1) \dot{v}>f(v)$ so $(-\dot{v})^{p-1}(p-1) \dot{v}>-v f(v)$ and integrating we obtain for $0<r<1$

$$
\frac{(p-1)}{p}(-\dot{v}(r))^{p}>\frac{(p-1)(-\beta)^{p}}{p}-\int_{\boldsymbol{r}}^{1} \dot{v}(t) f(v(t)) d t=\frac{(p-1)(-\beta)^{p}}{p}+\int_{\alpha}^{v(r)} f(s) d s .
$$

Thus,

$$
\begin{gathered}
-\dot{v}(r)>\left((-\beta)^{p}+\frac{p}{p-1} \int_{\alpha}^{v(r)} f(s) d s\right)^{1 / p}, \\
-\int_{1 / 2}^{1} \dot{v}(r)\left(\int_{\alpha}^{v(r)} f(s) d s\right)^{-1 / p} d r>\left(\int_{1 / 2}^{1}(-\beta)^{p}\left(\int_{a}^{v(r)} f(s) d s\right)^{-1}+\frac{p}{p-1}\right)^{1 / p} d r
\end{gathered}
$$

and

$$
\int_{\alpha}^{v(1 / 2)}\left(\int_{\alpha}^{t} f(s) d s\right)^{-1 / p} d t>\left(\int_{1 / 2}^{1}(-\beta)^{p}\left(\int_{\alpha}^{v(r)} f(s) d s\right)^{-1}+\frac{p}{p-1}\right)^{1 / p} d r .
$$

For fixed $\alpha$, it follows from (5) that the left hand side of (4.4) is bounded, independent of $\beta$. On the other hand, if $v(r)$ were to remain bounded with $-\beta$ large on the right-hand side, we would have a contradiction.

Having established $\beta<0$ so that (4.3) holds, we now apply (6) to show that there exists a value $r_{0}>1$ such that $\dot{v}(r) \rightarrow 0$ as $r \rightarrow r_{0}$.

Integrating the relation $\left((-\dot{v}(r))^{p-1} r^{n-1}\right)_{r}=-r^{n-1} f(v)$ we obtain for $r>1$

$$
(-\dot{v}(r))^{p-1} r^{n-1}=(-\beta)^{p-1}-\int_{1}^{r} t^{n-1} f(v(t)) d t .
$$

It follows that $r^{(n-1) /(p-1)} \dot{v}$ is increasing so for some $K>0$

$$
\begin{aligned}
v(r) & =\alpha+\int_{1}^{r} \dot{v}(t) d t=\alpha+\int_{1}^{r} \dot{v}(t) t^{(n-1) /(p-1)} t^{-(n-1) /(p-1)} d t \\
& \geqq \alpha+\beta \int_{1}^{r} t^{(1-n) /(p-1)} d t \geqq-K .
\end{aligned}
$$


We then have

$$
(-\dot{v}(r))^{p-1} r^{n-1}=(-\beta)^{p-1}-\int_{1}^{r} t^{n-1} f(v(t)) d t \leqq(-\beta)^{p-1}-f(-K) \int_{1}^{r} t^{n-1} d t,
$$

and the right-hand side will eventually be negative. Thus, $\dot{v}\left(r_{0}\right)=0$ for some $r_{0}>1$.

To summarize we have now shown that, given numbers $\alpha, M$, there exists $\beta=\beta(\alpha, M)$ such that if $v$ satisfies (4.1) and (4.2), then $v(1 / 2) \geqq M$ and there exists $r_{0}>1$, such that $v$ may be continued from $r=1$ to $r=r_{0}$, at which point $\dot{v}$ becomes 0 .

To complete the construction of $v$ past $r_{0}$ we continue by the equation

$$
v(r)=v\left(r_{0}\right)+\int_{r_{0}}^{r}\left(\frac{1}{t^{n-1}} \int_{r_{0}}^{t} s^{n-1} f(v(s))\right)^{1 /(p-1)} d t
$$

as in $\S 3$. Also as in $\S 3$ there exists $r_{1}>r_{0}$ such that $v(r) \rightarrow \infty$ as $r \rightarrow r_{1}$. parison.

With $v$ now completely described, the proof is now easily completed by com-

We may assume $S \subseteq|x|<1 / 4$. Suppose $u$ satisfies (4) outside $S$,

$$
M>\operatorname{ess~sup}_{1 / 4 \leqq|x| \leqq 1} u(x), \quad \alpha<\operatorname{essinf}_{1 / 2<|x|<3 / 2} u(x)
$$

and $v$ is the radial function previously constructed with $\beta=\beta(\alpha, M)$. Then, $v$ is $\mathscr{C}^{1}$ and satisfies (4.1) a.e.; hence $v$ is a radial solution to (4). But $v(r) \rightarrow \infty$ as $r \rightarrow r_{1}$, so by choice of $M$ and $\alpha$, Lemma A gives a contradiction. Hence $u$ cannot satisfy (4) in $\boldsymbol{R}^{n}-S$.

V. Some examples. Let $V(r)=\log \left((1+r)^{\beta} / r^{\gamma}\right),(\beta, \gamma>0)$. Then,

$$
\Delta_{n} V(r)=(n-1)\left|\frac{\gamma}{r^{2}}-\frac{\beta}{(1+r)^{2}}\right|^{n-2}\left(\frac{\beta}{r(1+r)^{2}}\right)
$$

so, for $\beta-\gamma<-2 n+1 \quad V$ is a radial subsolution $\Delta_{n} V \geqq e^{V}$ for $r$ sufficiently large. This shows that $p<n$ is needed in Theorem 2 (B). If $\gamma<2 n-3, V$ is a subsolution for sufficiently small $\varrho$ in $\{0<|x|<\varrho\}$.

Regarding Theorem 1, to show that $\delta>1$ is essential, we verify that the equation $\Delta_{n} u=e^{u}$ has a solution in some set $\{0<|x|<\varrho\}$, which is singular at $x=0$. In fact the radial form of $-\Delta_{n} u+e^{u}=0$ is the Euler equation for the functional $I[u]=$ $\int\left(\left|u_{n}\right|^{n}+n e^{u}\right) r^{n-1} d r$. For $\gamma<2 n-3$, let $V$ be a subsolution as above and $M_{r}=V(r)$ $(0<r<\varrho)$. The functional $I[u]$ has a minimizing function $u_{n}(r)$ [4; p. 24] on $[\varrho / n, \varrho]$ for each $n=2,3, \ldots$, with $u_{n}(\varrho)=M_{\varrho}, u_{n}(\varrho / n)=M_{\varrho / n}$. This $u_{n}(r)$ is a solution of the equation and by Lemma A $u_{n}(r) \geqq V(r)$ on $[\varrho / n, \varrho]$. To show that as $n \rightarrow \infty$ we obtain a solution with the desired properties, we need only bound the $u_{n}$ 's from above. To this end, let $\left\{\left|x-x_{0}\right| \leqq R\right\}$ be any closed ball in $\{0<|x|<\varrho\}$ and $n$ be sufficiently large so that it is contained in $\{\varrho|n<| x \mid<\varrho\}$. Let $v$ be the comparison function of $\S 1$, with $\delta=1, a=1$ in (1.4). Since $v(R)=\infty$, it follows from 
Lemma A that $u_{n} \leqq v$, for all sufficiently large $n$, in $\left\{\left|x-x_{0}\right|<R\right\}$. Thus, the $u_{n}$ 's are uniformly bounded in compact subsets of $\{0<|x|<\varrho\}$.

Finally, to see that condition (5) is sharp for Theorem 2(A), suppose $f$ is a positive nondecreasing locally Lipschitz function with

$$
\int_{0}^{\infty} d r\left(\int_{0}^{r} f(s) d s\right)^{-1 / p}=\infty .
$$

Then, there is a solution of (3.1) such that

$$
\dot{v}(0)=0, \dot{v}>0 \quad \text { if } \quad r>0, v \in \mathscr{C}^{1}[0, \infty] \cap \mathscr{C}^{2}(0, \infty) .
$$

Indeed, as in the proof of Lemma 2 we can construct a solution $v$ of (3.1) that will have the properties in (5.2) unless there is $r_{0}$ such that $\lim _{r \rightarrow r_{0}} v(r)=\infty$. We note that if this happens then (5.1) does not hold. For, since $\dot{v}(r)>0$ from (3.1) we obtain $\dot{v}^{(p-2)}((p-1) \ddot{v})<f(v)$. Hence, since without loss of generality we may assume $v(0)=0$, we have

$$
\int_{0}^{v(r)}\left(\int_{0}^{t} f(s) d s\right)^{-1 / p} d t<\left(\frac{p}{(p-1)}\right)^{1 / p} r .
$$

Making $r \rightarrow r_{0}$ we obtain a contradiction with (5.1).

On the other hand, it is not difficult to see that in fact we have

$$
\Delta_{p} v=f(v) \text { in } \mathscr{D}^{\prime}\left(\boldsymbol{R}^{n}\right) .
$$

VI. Concluding remark. In the case $\delta=1$, comparison of a solution of $\Delta_{n} u=e^{u}$ in $\{0<|x|<\varrho\}$ with the function $v$ of $\S 1$ yields $e^{u(x)} \leqq C /|x|$ for some $C>0$. Hence, $e^{u(x)} \in L_{n / n-\varepsilon} \quad(\varepsilon>0)$ and by [9; Theorem 1], if $u$ is a positive solution then $C_{1} \log (1 /|x|) \leqq u(x) \leqq C_{2} \log (1 /|x|)$. This estimate generalizes a theorem of Nitsche [5] for $n=2$.

\section{References}

[1] Bresis, H., and L. Véron: Removable singularities of some nonlinear elliptic equations. Arch. Rational Mech. Anal. 75 (1), 1980, 1-6.

[2] Keller, J.: On the solutions of $\Delta u=f(u)$. - Comm. Pure Appl. Math. 10, 1957, 503-510.

[3] Loewner, C., and L. Nirenberg: Partial differential equations invariant under conformal or projective transformations. - Contributions to Analysis, edited by L. V. Ahlfors et al., Academic Press, New York-London, 1974, 245-272.

[4] Morrey, C.: Multiple integrals in the calculus of variations. - Springer-Verlag, BerlinHeidelberg-New York, 1966.

[5] Nitsche, J.: Über die isolierten Singularitäten der Lösungen von $\Delta u=e^{u}$. Math. Z. 68, 1957, 316-324.

[6] Osserman, R.: On the inequality $\Delta u \geqq f(u)$. - Pacific J. Math. 7, 1957, 1641-1647.

[7] Redheffer, R.: On the inequality $\Delta u \geqq f(u,|\operatorname{grad} u|)$. - J. Math. Anal. Appl. 1, 1960, $277-$ 299.

[8] Serrin, J.: Local behavior of solutions of quasilinear equations. - Acta Math. 111, 1964. $247-302$. 
[9] SERRIN, J.: Isolated singularities of solutions of quasi-linear equations. - Acta Math. 113, $1965,219-240$.

[10] VASQUEZ, J. L.: On a priori interior estimate for the solutions of a non-linear problem representing weak diffusion. - Nonlinear Anal. 5, 1981, 95-103.

[11] VASQUEZ, J. L., and L. VÉRON: Removable singularities of some strongly nonlinear elliptic equations. - Manuscripta Math. 33, 1980, 129-144.

[12] Véron, L.: Singularites eliminables d'equations elliptiques non lineaires. - To appear.

[13] Walter, W.: Über ganze Lösungen der Differentialgleichungen $\Delta u=f(u)$. - Jber. Deutsch. Math.-Verein. 57, 1955, 94-102.

[14] Wiтtich, H.: Ganzer Lösungen der Differentialgleichung $\Delta u=f(u)$. - Math. Z. 49, 1944, $579-582$.

Purdue University

Department of Mathematics

West Lafayette, Indiana 47907

USA

Received 11 August 1981

Revision received 3 November 1981 